\title{
Cisj
}

\section{COMPUTER AIDED DIAGNOSIS USING SOFT-COMPUTING TECHNIQUES AND IMAGE'S ISSUED REPRESENTATION: APPLICATION TO BIOMEDICAL AND INDUSTRIAL FIELDS}

\author{
Kurosh Madani ${ }^{1)}$, Matthieu Voiry ${ }^{12}{ }^{2)}$, Véronique Amarger ${ }^{1)}$, Nadia Kanaoui ${ }^{1)}$, Amine \\ Chohra $^{1)}$, François Houbre ${ }^{2)}$
}

\author{
1) Images, Signals and Intelligent Systems Laboratory (LISSI / EA 3956), Senart Institute of Technology, \\ University PARIS XII, Av. Pierre Point, F-77127 Lieusaint, France \\ \{madani, amarger, chohra, kanaoui\}@univ-paris12.fr, http://www.univ-paris12.fr/ \\ 2) SAGEM REOSC, Avenue de la Tour Maury, Saint Pierre du Perray, 91280, France \\ \{mathieu.voiry, francois.houbre\}@sagem.com
}

\begin{abstract}
It is interesting to notice that from "problem's formulation" point of view "Industrial Computer Aided Diagnosis" and "Biomedical Computer Aided Diagnosis" could be formulated as a same diagnosis riddle: "How point out a correct diagnosis from a set of symptoms?". The only difference between the two above-mentioned groups of problems is the nature of the monitored (diagnosed) system: in the first group the monitored system is an artificial machinery (plant, industrial process, etc...), while in the second, the monitored system is a living body (animal or human).One of the most appealing classes of approaches allowing handling the Computer Aided Diagnosis Systems' design in the frame of the aforementioned dual point of view is Soft-Computing based techniques, especially those dealing with neural networks and fuzzy logic. In this article, we present two soft-computing based approaches dealing with CADS design. One aims designing a biomedical oriented CADS and the other sets sights on conceiving a CADS to overcome a real-world industrial quality control dilemma. The goal of the first system is to diagnose the human's auditory pathway's health. The target of the second is to detect and diagnose the high tech optical devices' defects.
\end{abstract}

Keywords: Computer aided diagnosis systems (CADS), soft-computing, artificial intelligent systems, industrial CADS applications, biomedical CADS applications.

\section{INTRODUCTION}

A "Computer Aided Diagnosis System" (CADS) is basically one which is able to identify (diagnose) the nature of a dysfunction by examining the observed symptoms. The output of such a system is called "diagnosis", defined as information identifying the type of faulty behavior. It may occur that diagnosis be complemented by additional information (such as an explanation or a justification) related to the pointed out diagnosis [1]. In a consequent number of CADS applications, it is desirable not only to identify the possible causes of the problem, but also to suggest suitable remedies (systems capable of advising) or to give a confidence rate of the possible causes' identification.

A Computer Aided Diagnosis System could be defined according to two different general points of view. The first one dissociates the faulty behavior's detection task from the diagnosis task. Thus in the frame of this point of view, a Computer Aided Diagnosis System requires three main stages: a first stage detecting the faulty behavior, a second stage classifying the faulty behavior and a last stage deciding the final diagnosis. While, the second point of view aggregates the detection and diagnosis tasks. According to this point of view, the pointed out diagnosis (information) includes the fact that the resulting diagnosis corresponds to a detected faulty behavior. According to this point of view, two stages are needed to achieve the diagnosis operation: a first stage identifying symptoms (classifying behavior, analyzing pertinent indicators, etc...) and a second deciding the appropriated diagnosis. Both of two above-mentioned CADS may include (when it is required) a number of additional stages (mainly one or two) performing a number of preprocessing tasks such as data preparation (normalization, data's adequate representation, etc...) or features' extraction (indicators, etc...).

Each of aforementioned frames corresponds to a number of advantages and disappoints. The main advantage of the CADS based on the first philosophy is to make available, independently from 
the resulted diagnosis, information on faulty healthy state of the monitored system. However, the need of a fault detection stage could be seen as a drawback making such CADS more costly to implement. The main advantage of the second slant comes from the fact that in such approaches (CADS) it is not necessary to detect the faulty behavior of the monitored system. The "healthy state" of the monitored system is considered as a possible pointed out diagnosis and thus a diagnosis stating any monitored system's faulty behavior means the presence (detection) of the corresponding fault. However, its main drawback is related to more complex information matching (more complex faults' models, more complex decision strategy, etc...).

Over past decades, several matching, classification and decision support systems taking advantage from bio-inspired Artificial Intelligence issued techniques have been developed [2], [3]. Recently a number of diagnosis approaches based on such intelligent artificial systems have been developed for industrial ([1], [4] and [5]) or biomedicine related purposes ([6], [7], [8], [9], [10] and [11]). It is interesting to note that from the point of view of the problem formulation "Industrial Computer Aided Diagnosis" (ICAD) and "Biomedical Computer Aided Diagnosis" (BCAD) could be formulated as a same diagnosis problem: to point out a diagnosis based on a number of symptoms. The only difference between the two above-mentioned groups of problems is the nature of the monitored system: in the first group the monitored system is an artificial machinery (plant, industrial process, etc...), while in the second, the monitored system is an alive body (animal or human).

One of the most used classes of approaches for feature identification, patterns' matching, classification and decision-making is soft computing based approaches, especially those dealing mainly with neural networks and fuzzy logic ([1], [3], [4], [5], [6], [9], [10], [11], [12], [13]). More recently, a number of research works dealing with abovementioned techniques leaded to a number of new soft-computing based solutions hybridizing those techniques aiming to solve real-world problems (complex systems' modeling, multiple criteria decision-making, fine classification, and nonlinear functions approximation) [14], [15], [16], opening new perspectives in CADS area.

In this article, we present two soft-computing based approaches dealing with CADS design. One aims designing a biomedical oriented CADS and the other sets sights on conceiving a CADS to overcome a real-world industrial quality control dilemma. The goal of the first system is to diagnose the human's auditory pathway's health. The target of the second is to detect and diagnose the high tech optical devices' defects.
The present paper is organized in following way: the next section will be dedicated to biomedical oriented CADS and related aspects. The first subsection of this section will introduce Auditory Brainstem Response (ABR) based clinical test. The second subsection of this second section will present the proposed CADS principle, its architecture and each of its three stages. The last sub-section of this section will give the obtained results. In section 3 of this paper we will present the industrial CADS and related investigations. The first sub-section of this section will introduce the problem to be solved, its industrial context and the proposed solution. The second sub-section will detailed the detection stage principle. The next sub-section will present preprocessing and feature extraction. Sub-section 4 will deal with an unsupervised learning based diagnosis issue. Finally, the last section will conclude the present article and discuss a number of perspectives.

\section{BIOMEDICINE ORIENTED HYBRID INTELLIGENT DIAGNOSIS APPROACH}

A wide class of medical diagnosis, as those performed by general practitioners in general medicine, is mainly based on deductive processes. The deductive processes involved in such medical diagnosis tasks are generally based on a number of symptoms leading to the issued diagnosis according to a deductive procedure as one of the following ones:

If symptom " $A$ " Then disease " $D$ "

If symptoms " $A$ " And " $B$ " And " $C$ " And ... Then disease " $D$ "

A number of works, over past decades, have suggested conventional "expert systems" as potential solution in CADS design for this class of medical diagnosis.

However, there exist in biomedicine a large number of cases where symptoms are not directly (or easily) discernable. In such cases, often, diagnosis is performed on the basis of clinical tests' results which are generally available either as signals (for example electroencephalograms [7], electrocardiograms [8], etc...) or as images (obtained, for example, from X-rays imagery, echographical imagery, IRN imagery, etc...). If, from a very general point of view, the procedure performed by the specialist physician to point out the diagnosis remains somewhere a deductive process, it could no more be formulated in the frame of the previously mentioned deductive schemes. In fact, the process leading to the final diagnosis in this second category of biomedical cases looks more like a pattern recognition process than a conventional deductive flow.

Concerning the CADS design relative to this second class of cases, the main difficulty remains the 
specialist physician reasoning flow's modeling which on the one hand is based on the expert's (specialist physician) deep experience, and on the other hand, takes advantage from complex (subjective, visual, etc...) information.

The present section deals with the design of a computer aided diagnosis system able to assert auditory pathologies using results issued from the Auditory Brainstem Response (ABR) based clinical test. ABR based clinical test takes advantage from Brainstem Auditory Evoked Potentials (BAEP) signals, which convey information related to hearing and brain (neurological) functioning [6], [17], [18], [19], providing an effective measure of the integrity of the auditory pathway. Based on what has been mentioned previously (concerning difficulty inherent to the expert's reasoning flow's modeling), the approach we adopt aggregates the detection and diagnosis tasks.

\subsection{BAEP SIGNALS AND ABR BASED CLINICAL TEST}

When a sense organ is stimulated, it generates a string of complex electrical responses related to its neurophysiology. BAEP are electrical response caused by the brief stimulation of a sense system. The stimulus gives rise to the start of a string of action's potentials that can be recorded on the nerve's course, or from a distance of the activated structures. The ABR clinical test, based on BAEP, is performed as follows (see Fig. 1): the patient hears clicking noise or tone bursts through earphones. The use of auditory stimuli evokes an electrical response. In fact, the stimulus triggers a number of neurophysiology responses along the auditory pathway. An action potential is conducted along the eight nerve, the brainstem, and finally to the brain. A few times after the initial stimulation, the signal evokes a response in the area of brain where sounds are interpreted [20].

Usually, the experts diagnose the pathology using a surface of 50 estimations called "Temporal Dynamic of the Cerebral" trunk (TDC) [20]. Figure 2 shows an example of TDC obtained for a healthy patient and a patient with harmful auditory disorder pathology, respectively.

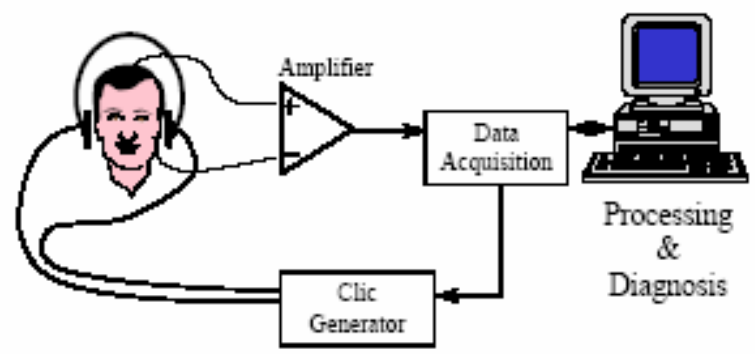

Fig. 1 - General bloc diagram of ABR clinical test chain.

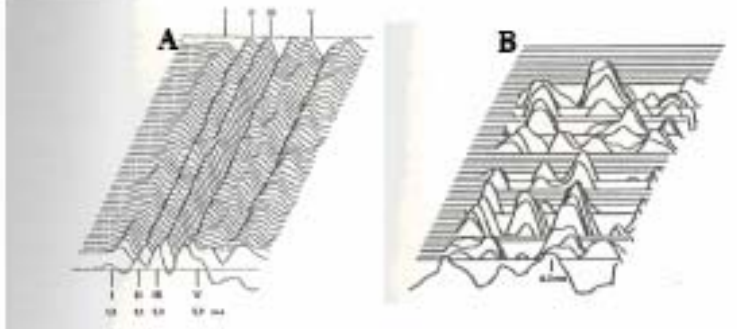

Fig. 2 - Examples of obtained TDC Surfaces showing a healthy (A) and an auditory disorder (B), respectively.

In general, for a healthy patient (normal audition), the ABR test leads to a regular TDC surface. However, it is not so easy to distinguish different TDC representations (surfaces) related to different type of pathologies. The results can vary for different test sessions for the same patient, because they depend on the person's relaxation, the background, the test's conditions, the signal-to-noise ratio, etc. Also, depending to the stage of auditory disorder, ABR test's results for two patients with different pathologies (for example one healthy and the second unwell) could look very similar. However, the expert (specialist physician) constructs his (or her) diagnosis from a visual analysis of the above-described TDC surfaces.

\subsection{PROPOSED CADS APPROACH}

Taking into account difficulty of modeling the expert's intellectual process leading to the final diagnosis, the suggestion is to design a CADS taking advantage of expert's knowledge (e.g. to use softcomputing approaches in order to learn from expert). Also, taking into consideration the expert's way to scrutinize the ABR clinical test's results, we have opted for an image-like representation of the CADS's input: the expert inspects TDC surfaces not as signals but looks at them as an image.

So, conformably to what has been mentioned in section 1 of this paper, the proposed CADS includes three stages: pre-processing stage, classification stage and decision stage.

The first stage converts the TDC surface to an image. The "Signal-To-Image" conversion is performed thanks to conventional threshold based interpolation techniques [21]. Fig. 3 depicts the bloc diagram of such transformation. As each BAEP signal is sampled and represented by 86 points (values) and a TDC surface includes 50 BAEP signals, consequently, each resulting image is formatted as a matrix of 50 rows and 86 columns. However, a finer analysis of these data leads us to consider a matrix of reduced dimensions: 40 rows by 80 columns. In fact, a number of last rows and first columns include many zero values and/or very high values as shown in Fig. 4 (black parts left-down). Finally, the reduced image is spitted into 16 subimages. 


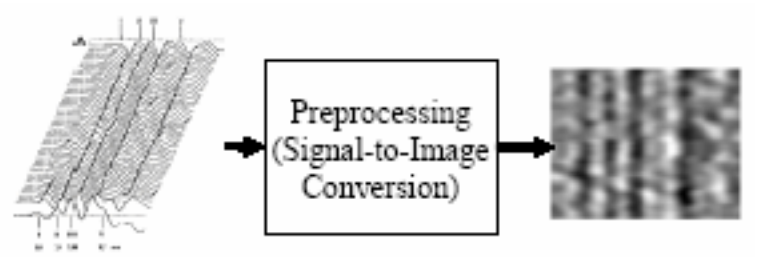

Fig. 3 - Bloc diagram of Signal-To-Image conversion.

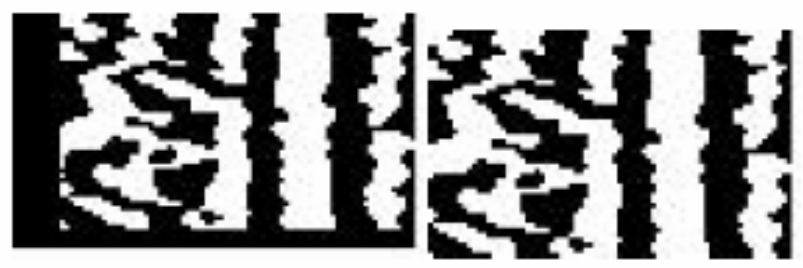

Fig. 4 - Example of TDC surface's image representation showing complete image (left) and reduced one (right), respectively.

The classification stage is based on a multiple neural networks structure [8], [12], [8], [9], [22]. It includes two kind of neural classifiers operating in an independent way: MLP and RBF [3], [14], [23], [24] and [25], as shown in Fig. 5. In this approach, 16 local sub-images (S_I-1, ..., S_I-i, ..., S_I-16) of each TDC issued image are classified within three classes, representing each a possible pathological category: "Normal Category Patient" (NCP: corresponding to a healthy patient), "Retro-cochlear Category Patient" (RCP: corresponding to a first possible pathological patient) and "Edo-cochlear Category Patient" (ECP: corresponding to a second possible pathological patient). The TDC image (e.g. all sub-images of a TDC issued image) is classified by both two kinds of the above-mentioned neural classifiers.

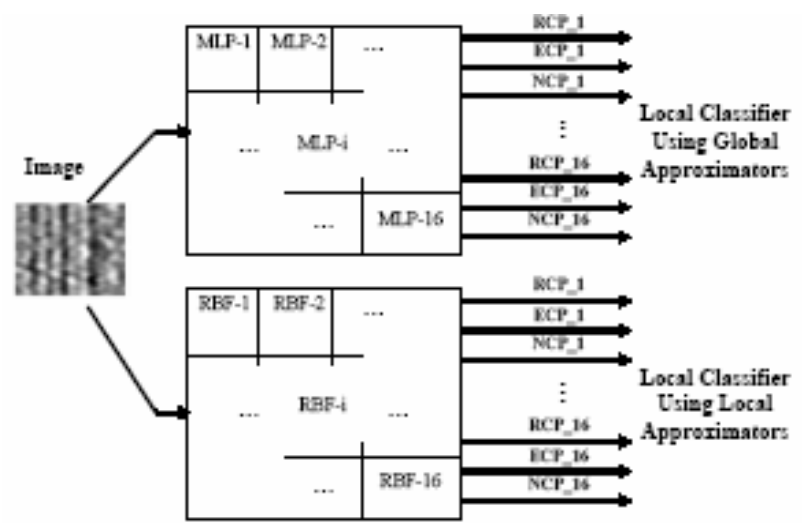

Fig. 5 - Synopsis of dual neural classification.

The last stage is composed of two fuzzy decisionmaking stages: primary Fuzzy System (FS 1) and final Fuzzy System (FS 2). Fig 6 shows the bloc diagram of the decision stage's architecture. Primary and final fuzzy decision-making stages consists of the Fuzzy System 1 (FS_1) and Fuzzy System 2 (FS_2), respectively, see Fig. 6. These fuzzy decision-making systems are used to capture the decision-making behavior of a human expert while giving the appropriate diagnosis [2], [3], [29] and [30].

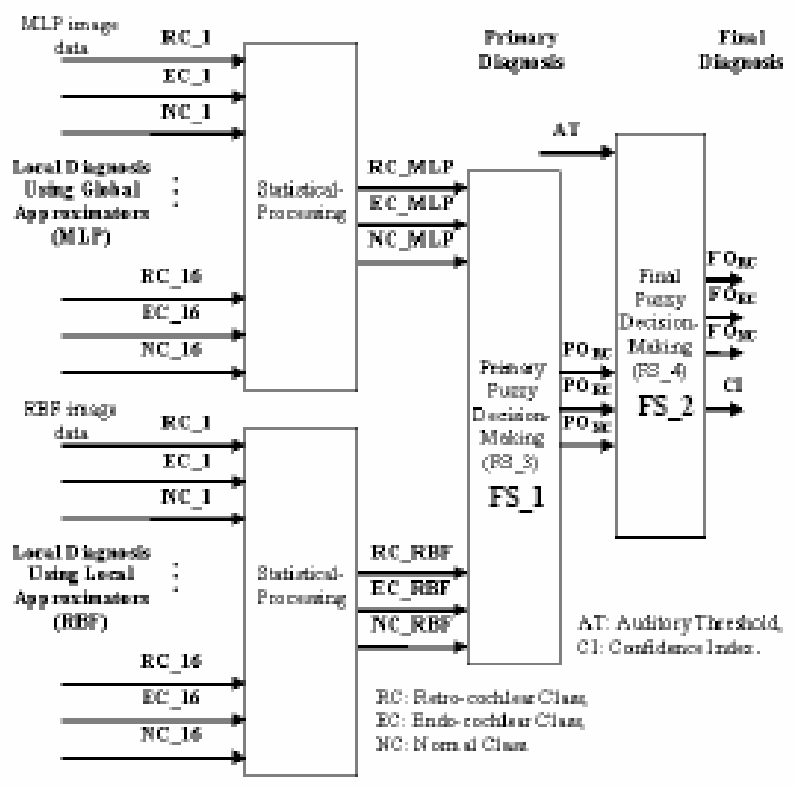

Fig. 6 - Synopsis of the hybrid fuzzy system based decision stage.

Note that both of two fuzzy inferences of FS 1 1 and FS 2 are Mamdani-like fuzzy inferences and are developed as detailed in [2], [3], [9], [26] and [27], with the simplification detailed in [28]. From this simplification, the fuzzy rule base of FS 1, which is built of $3^{6}=729$ rules, will operate only with $2^{6}=64$ rules in each inference. In the same way, the fuzzy rule base of FS_2, which is built of $3^{4}=81$ rules, will make in use only $2^{4}=16$ rules in each inference. Input parameters of FS 1, issued from the two neural classifiers, are RC MLP, EC MLP, NC_MLP, RC_RBF, EC_RBF, and NC_RBF. Its outputs are $\mathrm{PO}_{\mathrm{RC}}, \mathrm{PO}_{\mathrm{EC}}$, and $\mathrm{PO}_{\mathrm{NC}}$. The diagnosis' reliability obtained from FS 1 is reinforced (enhanced) using an additional parameter, called Auditory Threshold (AT). This parameter is exploited by FS_2 in order to generate the final diagnosis result. Input parameters of FS_2, issued from FS 1, are $\mathrm{AT}, \mathrm{PO}_{\mathrm{RC}}, \mathrm{PO}_{\mathrm{EC}}$, and $\mathrm{PO}_{\mathrm{NC}}$ and its outputs are $\mathrm{FO}_{\mathrm{RC}}, \mathrm{FO}_{\mathrm{EC}}$, and $\mathrm{FO}_{\mathrm{NC}}$ with their Confidence Index (CI).

\subsection{EXPERIMENTAL RESULTS}

The experimental results presented in this subsection have been obtained using a database containing data of 206 patients characterized by one of the three possible diagnoses (obtained from an expert). 206 images are built using this database leading to 38 images representing Retro-CochlearPatients, 77 images representing Endo-CochlearPatients, and 91 images representing NormalCochlear-Patients.

Table 1. Fuzzy decision-making system FS_1

\begin{tabular}{ccc} 
Results & Learning & Generalization (FS_1) \\
\hline RC & $100 \%$ & $10.52 \%$ \\
\hline EC & $100 \%$ & $15.78 \%$ \\
\hline NC & $100 \%$ & $77.77 \%$ \\
\hline
\end{tabular}


Table 2. Fuzzy decision-making system FS_2

\begin{tabular}{ccc} 
Results & Learning & Generalization (FS_2) \\
\hline RC & $100 \%$ & $21.05 \%$ \\
\hline EC & $94.87 \%$ & $57.89 \%$ \\
\hline NC & $100 \%$ & $82.22 \%$ \\
\hline
\end{tabular}

Obtained results are summarized in tables 1 and 2. Table 1 gives resulting pre-diagnosis performed by FS_1 decider. Table 2 gives final diagnosis results.

\section{INDUSTRIAL PRODUCTION ORIENTED INTELLIGENT CADS}

Fault diagnosis in industrial environment is a challenging but crucial task, since it ensures products' nominal specification and manufacturing control. Concerning High-Tech optical industry, a major step for high-quality optical devices' faults diagnosis concerns scratches and digs defects detection and characterization in such products. These kinds of aesthetic flaws, shaped during different manufacturing steps, could provoke harmful effects on optical devices' functional specificities, as well as on their optical performances by generating undesirable scatter light, which could seriously damage the expected optical features. A reliable diagnosis of these defects becomes therefore a crucial task to ensure products' nominal specification. Moreover, such diagnosis is strongly motivated by manufacturing process correction requirements in order to guarantee mass production quality with the aim of maintaining acceptable production yield. Figure 7 gives an example of High-Tech optical products, showing four optical filters.
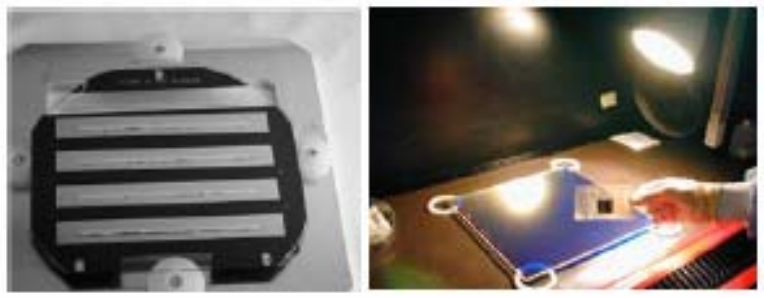

Fig. 7 - Example of High-Tech optical devices performing optical filtering (left) and the visual fault detection, performed by an expert.

Unfortunately, detecting and measuring such defects is still a challenging problem in production conditions and the few available automatic control solutions remain ineffective. That's why, in most of cases, the diagnosis is performed on the basis of a human expert based visual inspection of the whole production. However, this conventionally used solution suffers from several acute restrictions related to human operator's intrinsic limitations (reduced sensitivity for very small defects, detection exhaustiveness alteration due to attentiveness shrinkage, operator's tiredness and weariness due to repetitive nature of fault detection and fault diagnosis tasks).

\subsection{PROPOSED SOLUTION}

To construct an automatic diagnosis system, we propose an approach based on three main operations: detection, classification and decision. Our motivation to adopt the approach dissociating detection and diagnosis tasks is based on requirement relative to the frame of industrial production. In fact, two complementary options could de required in industrial production environment. The first is inherent to mass production where it is not always necessary to diagnose whole manufactured products during the production, but it is crucial to detect the presence of defects in order to state if the number of defects is conform to the process' intrinsic limitations. However, at the same time, diagnosis ability could help to state (offline) if detected defects are due to intrinsic limitations of the used manufacturing process or a number of them correspond to different derivations. The second situation is specific to HighTech products manufacturing requirements, where additionally to systematic defect detection it is crucial to state on nature of detected defects in order to reach high-quality specifications.

\subsection{DETECTION STAGE}

The detection stage is composed of two operations. The first operation is based on the Nomarski microscopy [29], [30], issued imaging (Nomarski microscope and a digital camera). The second operation incorporating the two following phases:

- Pre-processing: Nomarski microscopy issued digital image transformation in order to reduce lighting heterogeneity influence and to enhance the aimed defects' visibility,

- Adaptive matching: adaptive process to match defects,

is an image processing techniques based treatment of the Nomarski microscopy issued image in order to relieve defects' presence exposure. Fig. 8 gives the bloc diagram of the detection stage.

Three main advantages distinguishing Nomarski microscopy (known also as "Differential Interference Contrast microscopy" [30], [31] and [32]) from other microscopy techniques, have motivated our preference for this imaging technique. The first of them is related to the higher sensitivity of this technique comparing to the other classical microscopy techniques (Dark Field, Bright Field [29]). Furthermore, the DIC microscopy is robust regarding lighting non-homogeneity. Finally, this technology provides information relative to depth (3-rd dimension) which could be exploited to typify roughness or defect's depth. This last advantage 
offers precious additional potentiality to characterize scratches and digs flaws in high-tech optical devices. Therefore, Nomarski microscopy seems to be a suitable technique to detect surface imperfections. Issued images contain several items which have to be detected and then classified in order to discriminate between "false" defects (correctable defects) and "abiding" (permanent) ones. Indeed, because of industrial environment, a number of correctable defects (like dusts or cleaning marks) are usually present beside the potential "abiding" defects.

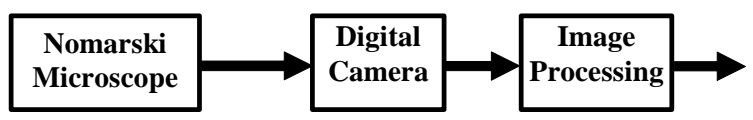

Fig. 8 - Bloc diagram of defects' detection chain.

In the pre-processing phase, as presented in [30] and [33], the intensity of every pixel in the image is modified according to the relation (1), where $P$ (respectively $P^{\prime}$ ) represents pixel's intensity before (respectively after) the transformation. $M$ and $\sigma$ are the mean and the standard deviation of grey-level in a $5 \times 5$ neighborhood of considered pixel.

$$
P^{\prime}=\frac{P}{M+\sigma}
$$
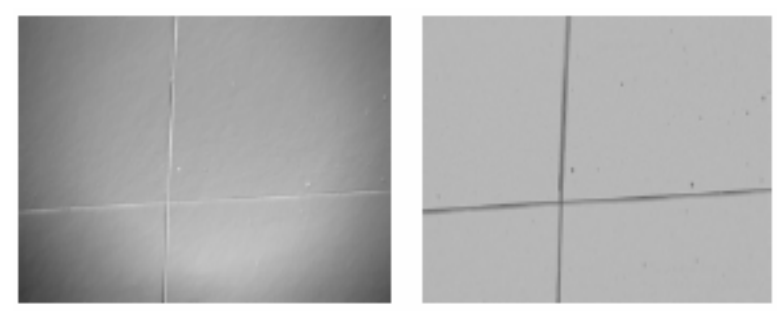

Fig. 9 - Example of pre-processing effects showing DIC microscopy issued defect's image (left) and the same captured defect after pre-processing phase.

The first consequence of the aforementioned transformation is to balance the image dynamic: lighting heterogeneities (due to non-uniform floodlighting) and local contrast modifications (due to focus and material thickness variations, or microscope optics imperfections) are corrected. Its second consequence is to enhance defects visibility in the processed image. Fig. 9 gives an example of a pre-processed DIC microscopy issued image, obtained conformably to the described transformation.

In order to perform defects detection, an adaptive thresholding operation is performed, exploiting physical considerations. To determine the effective threshold, we propose a technique based on the estimation of the roughness appearance. In our technique, the image (obtained after the preprocessing operation) is initially divided into a set of 8 by 8 pixels images. Then the number of 8 by 8 images in which the weakest available intensity corresponds to the same grey-level is determined. The evolution of the shape (curve) of this umber versus grey-level values is then determined. Finally, the effective adaptive threshold $\mathrm{T}$ is experimentally set thanks to relation (2), where Max is the greylevel corresponding to the maximum of the curve, and $H$ the WDMH (width of middle high) of the curve.

$$
T=M A X-\frac{H}{2}
$$

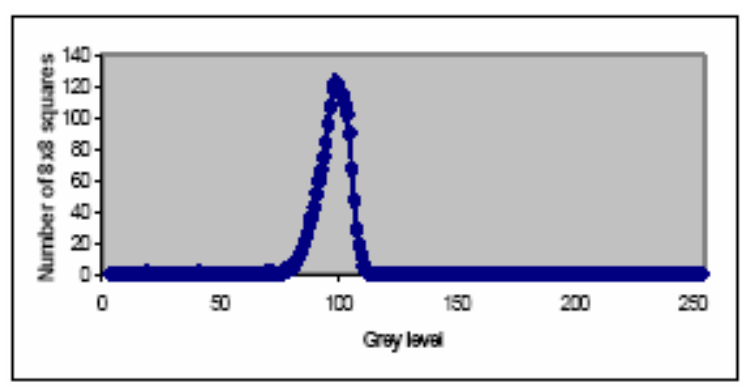

Fig. 10 - Example the curve expressing the number of 8 by 8 images in which the weakest available intensity corresponds to the same grey-level.

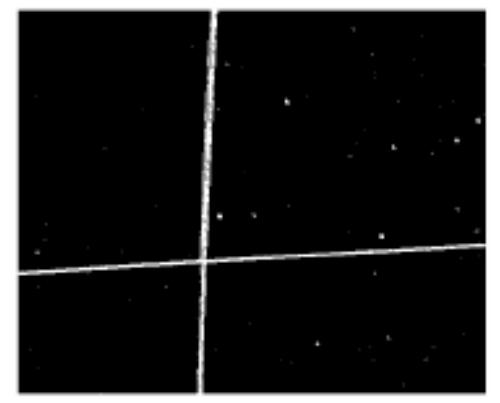

Fig. 11 - Example detection stage output image obtained from the DIC microscopy issued defect's image depicted in Fig. 9.

Fig. 10 gives an example of such curve for the images depicted by Fig. 9. An example of defect detection (e.g. detection stage's output image) is given in Fig. 11. It corresponds to resulted adaptive thresholding of the image shown in Fig. 9. The presented detection approach gives good and repetitive (robust) results, preserving sensitivity of DIC microscopy, since all of the defects deeper than roughness range are detected. Another interesting feature related to our approach is that the obtained curve is almost the same for any image.

\subsection{ITEMS IMAGES EXTRACTION}

The aim of this stage is to extract items images, taking into account DIC detector issued digital image, isolating different items. In shch way, an item (via its correponding image) will represent a pôtential defect to be diagnosed. To achieve this task, a new method is proposed, adding two new operations (phases) to the two previous ones (those described in detection stage):

- Filtering and segmentation: noise removal and defects' outlines characterization. 
- Defects' images extraction: correct defect representation construction.

The first operation consists of conventional filtering and morphological erosion transformation on image issued from the detection stage. It leads to a new image containing (representing) defects outlines. Filtering consists in replacing each pixel $\mathrm{P}$ by the $n^{\text {th }}$ pixel in the sorted list of all pixels in the m-square neighborhood of P. For little $n$ values, it is almost equivalent to a morphological dilatation but it filters isolated white pixels corresponding to noise. An example of resulted image, obtained using Fig. 11 results is given in Fig. 12.

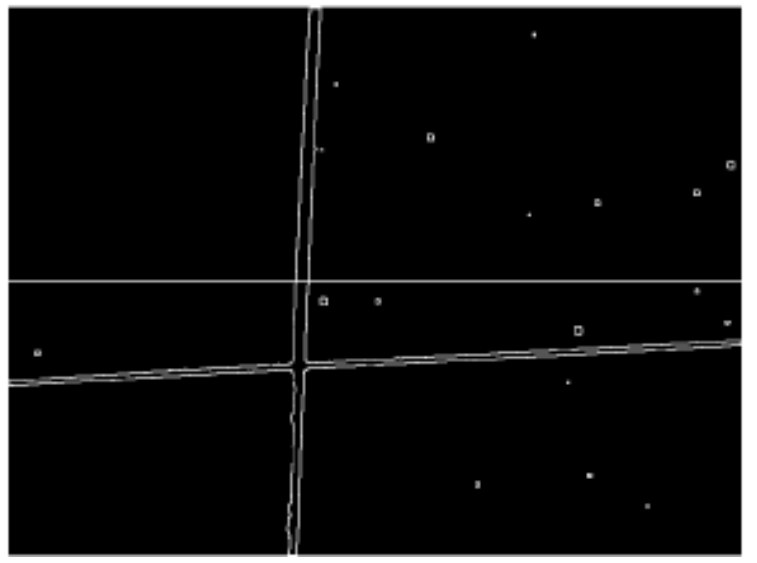

Fig. 12 - Example of resulted image obtained after the "filtering-segmentation" operation performed on image depicted in Fig. 11.

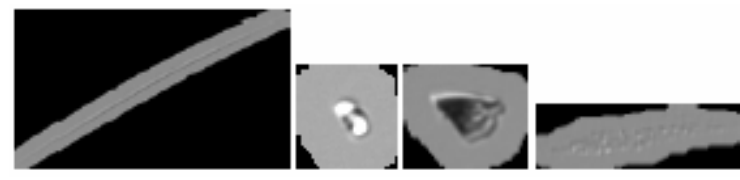

Fig. 13 - Examples of four detected items' images corresponding (from left to right) to "scratch-like", "dig-like", "dust-like" and "cleaning mark-like" defects.

Finally, images associated to all detected items are constructed performing the second operation. It is done considering a stripe of ten pixels around each "white" pixel of the detected item. Thus the obtained image gives an isolated (from other items) representation of the defect (e.g. depicts the defect in its immediate environment). Fig. 13 gives four examples of detected items images using the aforementioned principles, performed on raw images of an optical device in industrial environment. It shows different characteristic items which could be found on such device.

\subsection{DIAGNOSIS STAGE}

The diagnosis is performed by "clustering" (classification) then by comparison to the defects' categories' representative "specimens" (decision). The classification task is achieved thanks to an unsupervised learning based neural network which is a Kohonen Self-Organizing Map (SOM) [34] and [35]. However, before SOM based clustering, an additional operation is required. This additional operation consists on extracting a set of homogenous features in order to construct an invariant (regarding translation and rotation) representation of SOM's input vector. In fact, because of different sizes of items' images and their relative positions (due to translation and rotation) it is necessary to have a "normalize" representation for SOM's input patterns.

For that, we propose to use "Fourier-Mellin" transformation as it provides invariant descriptors, which are considered to have good coding capacity in classification tasks [36], [37] and [38]. A set of associated features, invariant with regard to geometric transformations, are proposed in [39]. In order to calculate efficiently Fourier-Mellin transform in discrete Cartesian coordinates, we perform the convolution of the image with an appropriate filters bench proposed in [42]. Finally, the extracted features have to be normalized. For this purpose we use the centring-reducing transformation modifying each feature $F_{i}$ conformably to the relation (3), where $M$ is the mean value of the feature $F_{i}$ over the database and $\sigma$ its standard deviation.

$$
F_{i}=\frac{F_{i}-M}{\sigma}
$$

\subsection{EXPERIMENTAL SET-UP AND VALIDATION RESULTS}

Three experiments called A, B, C were carried out, using two kind of optical devices (products). Table 3 shows the different parameters corresponding to these experiments. It's important to note that, in order to avoid false classes learning, items images depicting microscopic field boundaries or two (or more) different defects are discarded from used database.

Using the above described experimental protocol, we have diagnosed detected defects relative each above-mentioned experiment. Figures 14, 15 and 16 show the lattice of neurons corresponding to the three experiments respectively. In these figures, the depicted defect for each node is chosen randomly among the examples of the database which are projected onto the node under consideration. The size of images is normalized, so the real scale is not respected. In the three cases, the similarities between adjacent nodes are apparent and some clusters of similar data are identified. Moreover, in major cases, database items projected in the same neurons have the same appearance. Such defects probably belong to the same class of defects. Thus, the performed clustering operation seems relevant. However, data projected onto neurons which are near "natural" class boundaries, are sometimes inhomogeneous. 
Table 3. Experimental conditions and parameters

\begin{tabular}{cccccc}
$\begin{array}{c}\text { Exp. } \\
\text { Id. }\end{array}$ & $\begin{array}{c}\text { Optical } \\
\text { device } \\
\text { Id. }\end{array}$ & Clearing & $\begin{array}{c}\text { Number } \\
\text { scanned } \\
\text { Fields }\end{array}$ & $\begin{array}{c}\text { Number of } \\
\text { items in } \\
\text { learning }\end{array}$ & $\begin{array}{c}\text { SOM } \\
\text { grid size }\end{array}$ \\
\hline A & 1 & No & 1178 & 3865 & $15 \times 7$ \\
\hline B & 2 & No & 605 & 1910 & $20 \times 8$ \\
\hline C & 2 & Yes & 529 & 1544 & $10 \times 8$ \\
\hline
\end{tabular}

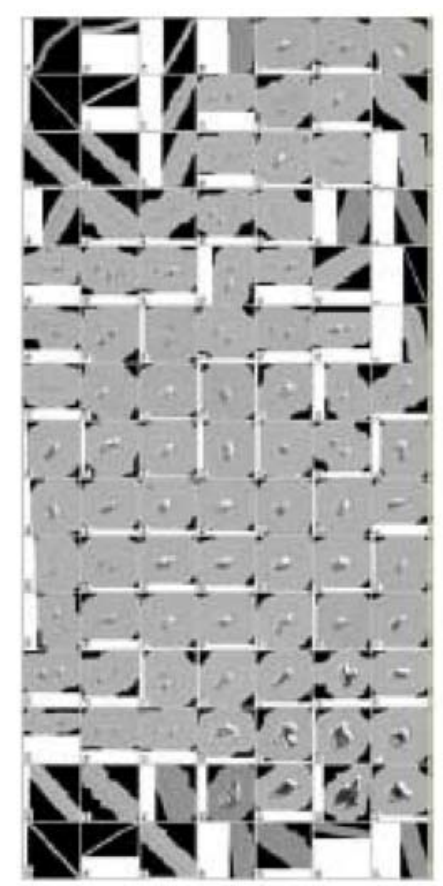

Fig. 14 - Representation of the Kohonen's map corresponding to the experiment $A$.

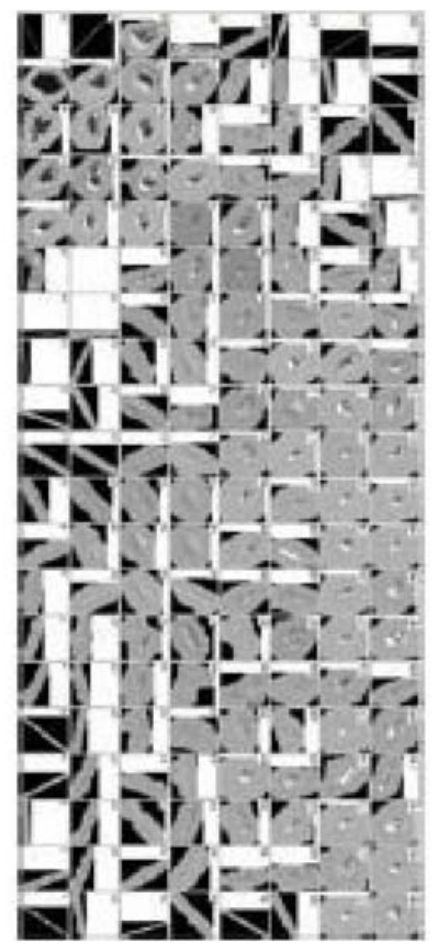

Fig. 15 - Representation of the Kohonen's map corresponding to the experiment $B$.

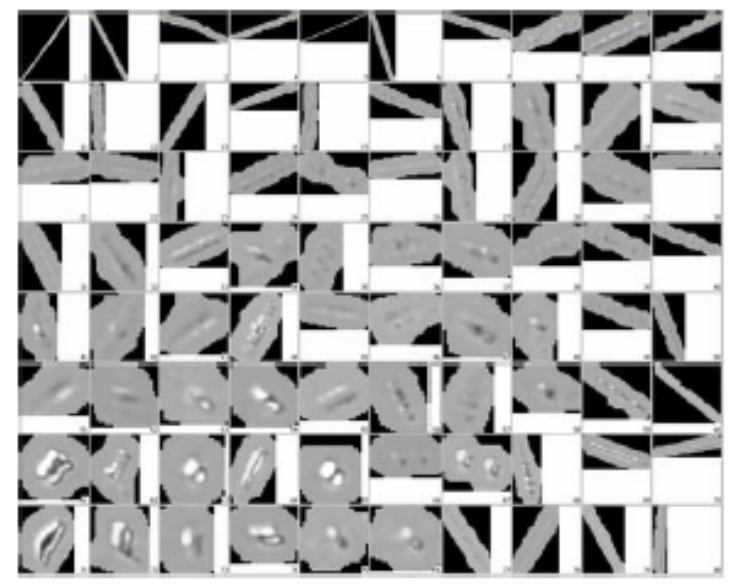

Fig. 16 - Representation of the Kohonen's map corresponding to the experiment $C$.

When observing obtained maps from experiments $\mathrm{A}$ and $\mathrm{B}$, we notice the presence of clusters similar in nature. On the contrary, when comparing maps from experiments $\mathrm{A}$ and $\mathrm{B}$ with the result of the experiment $\mathrm{C}$, we find that classes corresponding to big black items (see nodes 28, 29, 43, 44 from map A and nodes 17, 18, 19, 37, 38, 39 from map B) are absent in the third map. This is coherent with the fact that experiments $\mathrm{A}$ and $\mathrm{B}$ studied both non cleaned devices and therefore dealt with the same kind of defects, unlike experiment $C$. On the other hand, it implies that the mentioned defect classes would probably correspond to dusts.

\section{CONCLUSION}

Taking into consideration the fact that "Industrial Computer Aided Diagnosis" and "Biomedical Computer Aided Diagnosis" could be regarded as a same diagnosis riddle, we have presented two CADS, taking advantage from the most appealing abilities of ANN which are their "learning", "generalization and for some of them their "selforganization" capabilities. Both of the two Computer Aided Diagnosis Systems operate on the basis of image-like information's representation, covering a large number of real-world applications either in biomedicine or in industry.

Based on a neural classifiers' fusion (involving two different kind of neural networks) and a cascaded fuzzy decision-making strategy, the biomedical oriented CADS has been applied to overcome the difficult challenge of human's auditory disorders' diagnosis. While the target of the second, based on combination of a defects' detection stage and a Kohonen-SOM-like unsupervised ANN, was to detect and diagnose the defects in High-Tech optical devices in the frame of industrial production constraints.

The obtained experimental validation results, carried out using real data, as well for the biomedical application as for the industrial request, show the promising potential of Soft-Computing techniques in designing intelligent CADS. 
Further works, dealing with the present paper's purposes, are already engaged in several directions. Part of them is oriented on mechanical faults detection and diagnosis in industrial power machines. Another slice of planed perspectives concerns other kinds of digital imaging based CADS applications in industry. Also, a part of perspectives launch new clinical applications.

\section{REFERENCES}

[1] K. Balakrishnan, V. Honavar, Intelligent Diagnosis Systems, Tech. Report, Iowa State Univ., Ames, Iowa 50011-1040, U.S.A., 1997.

[2] E. Turban, J.E. Aronson, Decision Support Systems and Intelligent Systems, Int. Edition, Sixth Edition, Prentice-Hall, 2001.

[3] F. O. Karray , C. De Silva, Soft Computing and Intelligent Systems Design, Theory, Tools and Applications, Addison Wesley, ISBN 0-32111617-8, 2004.

[4] M. Meneganti, F. S. Saviello, R. Tagliaferri, Fuzzy Neural Networks for Classification and Detection of Anomalies. IEEE Transact. on Neural Networks, 9, No. 5, pp. 848-861, 1998.

[5] G. I. S. Palmero, J. J. Santamaria, E. J. M. de la Torre, J. R. P. Gonzalez, Fault Detection and Fuzzy Rule Extraction in AC Motors by a Neuro-Fuzzy ART-Based System. Engineering Applications of AI, 18, Elsevier, pp. 867-874, 2005.

[6] J. H. Piater, F. Stuchlik, H. Von Specht, R. Mühler, Fuzzy Sets for Feature Identification in Biomedical Signals with Self-Assessment of Reliability: An Adaptable Algorithm Modeling Human Procedure in BAEP Analysis. Comput. and Biomedical Resear., 28, pp. 335-353, 1995.

[7] A. Vuckovic, V. Radivojevic, A. C. N. Chen, D. Popovic, Automatic Recognition of Alertness and Drowsiness from EEG by an Artificial Neural Network. Medical Engineering \& Physics, 24 (5), pp. 349-360, 2002.

[8] A. Wolf, C. H. Barbosa, E. C. Monteiro, M. Vellasco, Multiple MLP Neural Networks Applied on the Determination of Segment Limits in ECG Signals. LNCS 2687, SpringerVerlag Berlin Heidelberg, pp. 607-614, 2003.

[9] A. Chohra, N. Kanaoui, V. Amarger, A Soft Computing Based Approach Using Signal-ToImage Conversion for Computer Aided Medical Diagnosis (CAMD). Information Processing and Security Systems, Ed.: K. Saeed, J. Pejas, Springer, pp. 365-374, 2005.

[10]A. Chohra, N. Kanaoui, K. Madani, Hybrid Intelligent Classification for Computer Aided Diagnosis (CAD) Systems Using Image Representation. Int. Journal Image Processing and Communications, Vol. 10, No. 2, ISSN 1425-140x, pp. 07-15, 2005.

[11]H. Yan, Y. Jiang, J. Zheng, C. Peng, Q. Li, A Multilayer Perceptron-Based Medical Support System for Heart Disease Diagnosis. Expert Systems with Applications, Elsevier, 2005.

[12]R. Murray-Smith, T.A. Johansen, Multiple Model Approaches to Modelling and Control, Taylor \& Francis Publishers, 1997.

[13] J. Kittler, M. Hatef, R. P. W. Duin, J. Matas, On Combining Classifiers. IEEE Trans. Pattern Analysis and Machine Int., Vol. 20, No. 3, pp. 226-239, 1998.

[14] S. Haykin, Neural Networks: A Comprehensive Foundation, 2Ed. Prentice-Hall, 1999.

[15]G. P. Zhang, Neural Networks for Classification: A Survey. IEEE Trans. on Systems, Man, and Cybernetics, Vol. 30, no. 4, 451-462, 2000.

[16] M. Egmont-Petersen, D. De Ridder, H. Handels, Image Processing with Neural Networks - A Review. Pattern Recognition, 35, pp. 22792301, 2002.

[17]M. Don, A. Masuda, R. Nelson, D. Brackmann, Successful Detection of Small Acoustic Tumors using the Stacked Derived-Band Auditory Brain Stem Response Amplitude. The American Journal of Otology 18, 5, pp. 608-621, 1997.

[18]E. Vannier, O. Adam, J.F. Motsch, Objective Detection of Brainstem Auditory Evoked Potentials with a Priori Information from Higher Presentation Levels. Artificial Intelligence in Medicine, Vol. 25, pp. 283-301, 2002.

[19]A. P. Bradley, W. J. Wilson, On Wavelet Analysis of Auditory Evoked Potentials. Clinical Neurophysiology, 115, pp. 1114-1128, 2004.

[20] J. F. Motsh, La dynamique temporelle du tronc cérébral: receuil, extraction, et analyse optimale des potentiels évoqués auditifs du tronc cérébral, $\mathrm{PhD}$ Thesis, Paris-XII University, 1987 (in French).

[21]R. C. Gonzalez and R. E. Woods, Digital Image Processing, 2 Ed., Prentice-Hall, 2002.

[22] K. Madani, L. Thiaw, R. Malti, G. Sow, MultiModeling: a Different Way to Design Intelligent Predictors, LNCS 3512: "Computational Intelligence and Bio-inspired Systems”, Ed.: J. Cabestany, A. Prieto, and F. Sandoval, Springer Verlag Berlin Heidelberg, ISBN 3-540-26208-3, pp. $976-984,2005$

[23]L.M. Reyneri, Weighted Radial Basis Functions for Improved Pattern Recognition and Signal Processing, Neural Processing Let., Vol. 2, No. 3, pp 2-6, May, 1995.

[24] G. Trémiolles (de), K. Madani, P. Tannhof, A New Approach to Radial Basis Function's like 
Artificial Neural Networks, NeuroFuzzy'96, IEEE European Workshop, Vol. $6 N^{\circ} 2$, pp 735745, April 16 to 18, Prague, Czech Republic, 1996.

[25] M.A. Arbib (ed.), Handbook of Brain Theory and Neural Networks, 2ed. M.I.T. Press. , 2003.

[26] O, Azouaoui, A. Chohra, Soft Computing Based Pattern Classifiers for the Obstacle Avoidance Behavior of Intelligent Autonomous Vehicles (IAV). Int. J. of Applied Intelligence, Kluwer Academic Publishers, 16, no. 3, pp. 249-271, 2002.

[27]L. A. Zadeh, The Calculus of Fuzzy If / Then Rules. AI Expert, 23-27, 1992.

[28]H. Farreny, H. Prade, Tackling Uncertainty and Imprecision in Robotics, $3^{\text {rd }}$ Int. Symp. on Robotics Research, pp. 85-91, 1985.

[29]P. E. J. Flewitt and R. K. Wild, Light Microscopy. Physical Methods for materials characterisation, 1994.

[30] M. Voiry, F. Houbre, V. Amarger, and K. Madani, Toward Surface Imperfections Diagnosis Using Optical Microscopy Imaging in Industrial Environment, Workshop on Advanced Control and Diagnosis, Mulhouse, France, pp. 139-144, 2005.

[31]P. Bouchareine, Métrologie des Surfaces. Techniques de l'Ingénieur, vol. R1390, 1999 (in French).

[32] S. Chatterjee, Design Considerations and Fabrication Techniques of Nomarski Reflection Microscope. Optical Engineering, vol. 42, no. 8, pp. 2202-2212, 2003.

[33] M. Voiry, K. Madani, V. Amarger, F. Houbre, Toward Automatic Defects' Clustering in Industrial Production Process Combining Optical detection and Unsupervised Artificial Neural Network techniques, Artificial Neural Networks and Intelligent Information Processing, INSTICC Press, N${ }^{\circ}$ ISBN: 978-9728865-68-9, pp. 25-34, 2006.

[34] T. Kohonen, E. Oja, O. Simula, A. Visa, and J. Kangas, Engineering Applications of the SelfOrganizing Maps. Proceedings of the IEEE, vol. 84, no. 10, pp. 1358-1384, 1996.

[35] T. Kohonen, Self Organizing Maps, 3rd edition, Berlin: Springer, 2001.

[36] A. Choksuriwong, H. Laurent, and B. Emile, Comparison of invariant descriptors for object recognition. IEEE International Conference on Image Processing (ICIP) pp. 377-380, 2005.

[37] S. Derrode, Représentation de Formes Planes à Niveaux de Gris par Différentes Approximations de Fourier-Mellin Analytique en vue d'Indexation de Bases d'Images. Phd Thesis Rennes I Univ., 1999. (in French)

[38]F. Ghorbel, A Complete Invariant Description for Gray Level Images by the Harmonic Analysis Approach. Pattern Recognition, vol. 15, pp. 1043-1051, 1994.

[39] G. Ravichandran and M. Trivedi, CircularMellin features for texture segmentation. IEEE Trans. Image Processing, vol. 4, pp. 1629-1640, 1995.

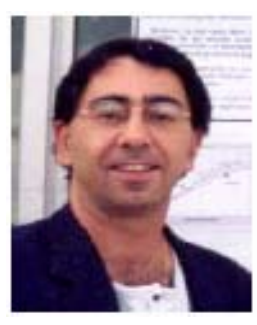

Prof. Kurosh Madani. Received his Ph.D. degree in Electrical Engineering and Computer Sciences from University PARIS XI, Orsay, France, in 1990. From 1989 to 1990, he worked as assistant professor at Institute of Fundamental Electronics of PARIS XI University. In 1990, he joined CreteilSenart Institute of Technology of University PARIS XII - Val de Marne, Lieusaint, France, where he worked from 1990 to 1998 as assistant professor. In 1995, he received the DHDR Doctor Habilitate degree (senior research Dr. Hab. degree) from University PARIS XII - Val de Marne. Since 1998 he works as Chair Professor in Electrical Engineering of Senart Institute of Technology of University PARIS XII. From 1992 to 2004 he has been head of Intelligence in Instrumentation and Systems Laboratory (I2S / JE 2353) located at Senart Institute of Technology. Since 2005, he is head of one of the three research groups of Image, Signal and Intelligent Systems Laboratory (LISSI / EA 3956) of PARIS XII University. He has worked on both digital and analog implementation of processors arrays for image processing, electro-optical random number generation, and both analog and digital ANN implementation. His current research interests include large ANN structures modeling and implementation, hybrid neural based information processing systems and their software and hardware implementations, design and implementation of real-time neuro-control and neural based fault detection and diagnosis systems. Since 1996 he is a permanent member (elected Academician) of International Informatization Academy. In 1997, he was also elected as Academician of International Academy of Technological Cybernetics.

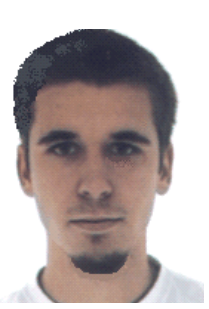

Matthieu Voiry. Graduated in 2002 from ENSEIRB and received his MS degree in 2003 from Bordeaux 2 University. Currently he is working toward his Ph.D. at Image, Signal and Intelligent Systems Laboratory (LISSI / EA 3956) of PARIS XII University with Prof. Kurosh Madani, in collaboration with SAGEM REOSC Company. His main research interests are image processing and artificial neural networks in industrial computer aided diagnosis. 


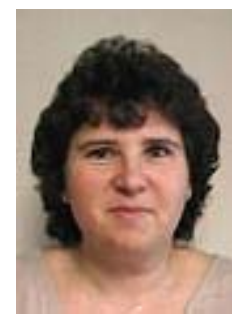

Dr. Véronique Amarger. Received her PhD degree in Microelectronics and Computer Science from the University of Paris 7, France, in 1993. Since 1993, she has joined Senart Institute of Technology of PARIS XII - Val de Marne University, one of two Institutes of Technology of this University, where she works as Assistant Professor. She is a Staff Member of Image, Signal and Intelligent Systems Laboratory (LISSI / EA 3956) of PARIS XII University. Her main research interests concern the field of Bio-inspired Artificial Intelligence, Neural Networks and Computer Aided Diagnosis Systems design and applications.

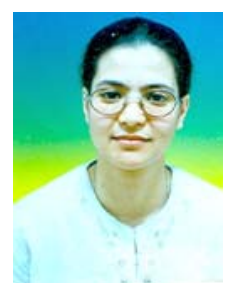

Nadia Kanaoui. Received her Engineer degree in Electrical Engineering and Computer sciences in 2000 from Sciences and Technologies Faculty of Settat Unicersity, Settat (Morocco). She received her MS in Computer Sciences from Ecole Polytechnique de Nantes, Nantes (France) 2002. Since 2002 she is preparing a Ph.D. in Computer Sciences at Image, Signal, and Intelligent Systems Laboratory (LISSI / EA 3956) of PARIS XII University (France). Her research interests are related to hybrid computer aided diagnosis, image processing, classification, decision-making, neural networks and fuzzy approaches.

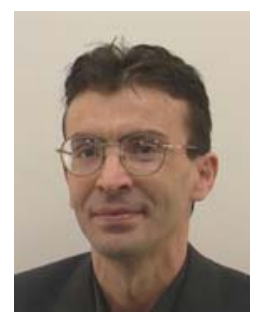

Dr. Amine Chohra. Received his Doctorate es-sciences in 1999 from Ecole Nationale Polytechnique, Algiers (Algeria). He has been a member of Artificial Intelligence and Robotics Laboratory (LRIA) of CDTA from 1991 to 1999. From 1999 to 2001, he worked as post doctoral researcher, with Behavior Engineering team of AiS-GMD, Sankt
Augustin (Germany) and with Dependable Computing Group of IEI-CNR, Pisa (Italy), respectively. From 2001 2003, he has been teacher/researcher at Orleans University, ENSI de Bourges (France), and a member of Vision and Robotics Laboratory (LVR / UPRES EA 2078). Since September 2003, he is Assistant Professor at Senart Institute of Technology of PARIS XII University, Lieusaint (France) and a staff member of Image, Signal, and Intelligent Systems Laboratory (LISSI / EA 3956) of this University. His research interests are information processing systems, knowledge based systems, hybrid intelligent systems, soft computing, machine learning and decision-making, pattern recognition and computer aided diagnosis.

Mr. François Houbre. Graduated in 1990 from ESO (Ecole Supérieure d'Optique d'Orsay, France). He worked 8 years in optical engineering team of Sfim Group with different functions as System Engineer and optical workshop manager. Then, he joint

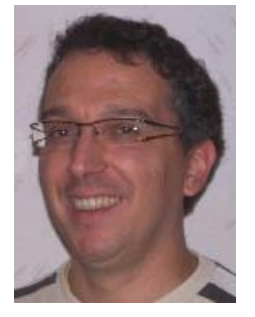
SAGEM REOSC team in 1998 as Project Manager and further product line Manager. He is now managing since 2004 the "process and instrumentation" group of Saint Pierre du Perray $R \& D$ Center. 